\title{
Flow distribution in diverging compound channels using improved independent subsection method
}

\author{
Bhabani Shankar Das ${ }^{1,}$, Kamalini Devi ${ }^{1}$, Sebastien Proust $^{2}$ and Kishanjit Kumar Khatua ${ }^{1}$ \\ ${ }^{1}$ National Institute of Technology Rourkela, Civil Engineering Department, 769008 Rourkela, India \\ ${ }^{2}$ Irstea, UR RiverLy, centre de Lyon-Villeurbanne, 69625 Villeurbanne, France
}

\begin{abstract}
Experiments have been conducted in three diverging compound channels for different flow conditions to study the flow distribution in floodplain, upper and lower main channel. In a compound channel, vertical apparent shear exists on the interface between the upper main channel and the floodplain, which generally accelerates the flow on the floodplain and resists the flow in the upper main channel. In addition, a horizontal apparent shear stress also occurs on the interface between the upper and lower main channels, which generally accelerates the flow in the lower one and resists the flow in the upper one. Therefore, it is essential to consider the exchanges of momentum at both vertical and horizontal shear layer regions. In this paper, an attempt is made to improve the classical independent subsection method (ISM) to determine the magnitudes of flow and velocities in both upper and lower main channels. Four subsections are created in improved ISM according to the vertical and horizontal division lines that correspond to the vertical interface between the main channel and floodplain and the horizontal interface between upper and lower main channels respectively. The improved ISM consists in a set of four coupled 1D momentum equations (instead three equations of classical ISM) for subsections and a mass conservation equation for the total cross-section. The computed results show that the method is well capable of predicting the discharge distributions in the floodplain and main channel (both at upper and lower main channel).
\end{abstract}

\section{Introduction}

Flow modelling in the compound channel is challenging task for the river engineer. When the geometry of floodplains diverges or converges, that affect significantly to the conveyance estimation. Flooding rivers usually present flow-width variations that give rise to non-uniform flows in non-prismatic compound geometries. Flow distribution in the diverging compound channel is a very important topic in river hydraulics to be investigated from a practical point of view in relation to flood risk assessment, bank protection, navigation and sediment-transport depositional pattern. Knowledge on velocity distribution

\footnotetext{
* Corresponding author: bsd.nitrk1@gmail.com
} 
in a compound channel (main channel and floodplains) helps to determine the energy expenditure, bed shear stress distribution, and the associated mass and momentum transport problems. Very few works found from the literature on a compound channel with diverging floodplains. Proust [1], is the first to worked on diverging compound channels and presented an independent subsection method to model the flow depth and velocity at the different subsections of prismatic and non-prismatic compound channels. Bousmar et al. [2] discuss the flow behaviour in the compound channel with diverging floodplain for two different diverging angles $3.81^{\circ}$ and $5.7^{\circ}$. Later Yonesi et al. [3] worked on diverging compound channels with differential bed roughness for diverging angles $3.81^{\circ}, 5.7^{\circ}$ and $11.3^{\circ}$. Due to non-prismatic effect of the floodplain the existing traditional methods like single channel method (SCM), divided channel method (DCM) and numerical methods like lateral distribution method (LDM), Shiono and Knight method (SKM) are failed to provide accurate stage, discharge, and velocity at different sections of the non-prismatic portion [4, $5,6]$.

Knight and Demetriou [7], Wormleaton et al. [8] and Devi et al. [9] show that the vertical apparent shear exists on the interface between the upper main channel and the floodplain, which generally accelerates the flow on the floodplain and resists the flow in the upper main channel. In addition, a horizontal apparent shear stress also occurs on the interface between the upper and lower main channels, which generally accelerates the flow in the lower one and resists the flow in the upper one [10]. Therefore, it is essential to consider the exchanges of momentum at both vertical and horizontal shear layer regions. Now coming to the existing numerical methods, the Independent Subsection Method, ISM, which was developed to model streamwise non-uniform flows for both prismatic and nonprismatic compound channels was found to give good results of water depth and velocity distribution in main channel and floodplain [11,12] and also depth-averaged Reynolds stress at the vertical interface main channel / floodplain [13]. In this method, a1D momentum equation is solved in each of the three subsections (left floodplain, main channel, right floodplain) and a continuity equation for the whole section. Thus, this method constitutes four equations and solves simultaneously to get the mean velocity and water depth at all sub-sections. With a vertical division of the sub-sections, the method does not consider the momentum transfer between the upper and lower parts of the main channel. An attempt is made here to improve the ISM to determine the magnitudes of flow and velocities in the upper and lower main channels. For this, experiments have been conducted in three different diverging angles to measure the lower and upper main channel discharge and the calibrating coefficient which affects the simulation of ISM.

\section{Experimental setup}

Experiments on a compound channel having diverging floodplains have been performed at the Hydraulics Engineering Laboratory of National Institute of Technology, Rourkela, India. Three sets of compound channels with diverging floodplains made up of perspex sheet were fabricated inside a tilting flume of size $22 \mathrm{~m}$ long $\times 2 \mathrm{~m}$ width $\times 0.5 \mathrm{~m}$ depth. Keeping the geometry of main channel constant, the diverging length of the fabricated channels were changed to $5 \mathrm{~m}, 3 \mathrm{~m}$, and $2 \mathrm{~m}$. The diverging angles of the floodplains were estimated to be $5.93^{\circ}, 9.83^{\circ}$, and $14.57^{\circ}$ respectively. Longitudinal bed slope of the channel was maintained at $1.40 \times 10^{-3}$, satisfying subcritical flow conditions. The roughness of the floodplain and main channel were alike and the Manning's $n$ found out as 0.011 from the in-bank experimental runs in the channel. In order to compare the results of experiments in non-prismatic compound channels with different divergence angles $(\theta)$ for each selected discharge, the downstream water level was adjusted using the tailgate. It was done in such a 
way that the backwater profile reached a given depth in the central section of diverging reach at $\mathrm{x}=14.5 \mathrm{~m}, \mathrm{x}=15.5 \mathrm{~m}$ and $\mathrm{x}=16 \mathrm{~m}$ for $5 \mathrm{~m}, 3 \mathrm{~m}$ and $2 \mathrm{~m}$ diverging portions of the compound channels respectively (Fig. 1). Water depths were measured directly with a point gauge located on an instrument carriage. A micro-Pitot tube of $4.77 \mathrm{~mm}$ external diameter in combination with suitable inclined manometers as well as $16-\mathrm{MHz}$ Micro-ADV (Acoustic Doppler Velocimeter) were used to measure velocities. Details of experimental setup and procedures are available in Das and Khatua [6, 14]. Summary of experimental characteristics of present test channels are given in Table 1.
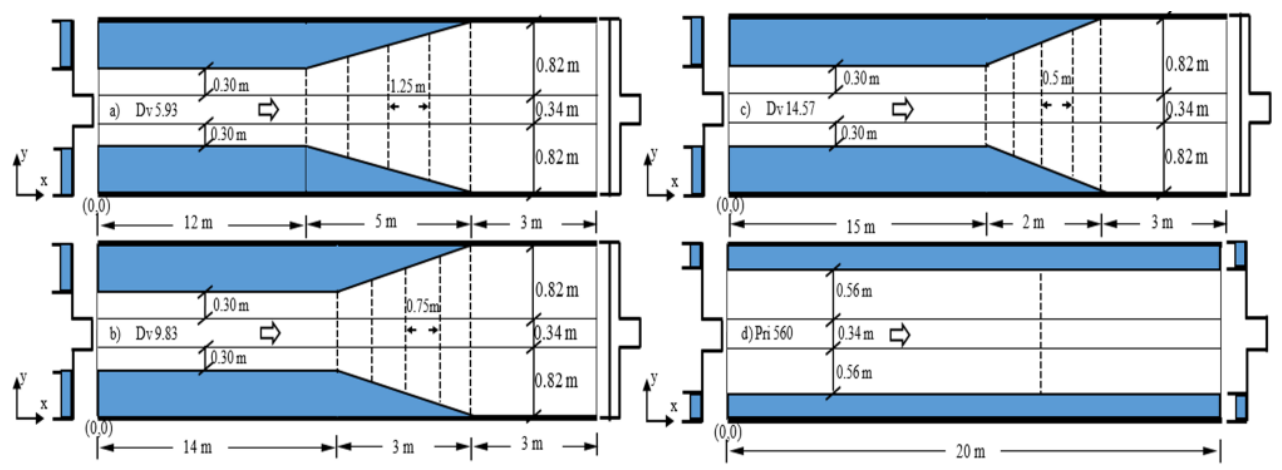

Fig. 1. Schematic view of compound channels with non-prismatic floodplains, diverging from $300 \mathrm{~mm}$ to $820 \mathrm{~mm}$ along a length of (a) $5 \mathrm{~m}$ (Dv 5.93), (b) $3 \mathrm{~m}$ (Dv 9.83), and (c) $2 \mathrm{~m}$ (Dv 14.57) and a prismatic compound channel with $560 \mathrm{~mm}$ floodplain width (d)Pri 560

Table 1. Summary of experimental characteristics

\begin{tabular}{|c|c|c|c|c|c|}
\hline Series & Geometry & $\begin{array}{c}\text { Relative flow } \\
\text { depth, } \mathbf{D r}^{(\mathbf{1})}\end{array}$ & Discharge $\mathbf{Q}\left(\mathbf{m}^{\mathbf{3}} / \mathbf{s}\right)$ & Fr & $\begin{array}{c}\mathbf{R e} \\
\left(\times \mathbf{1 0}^{\mathbf{5}}\right)\end{array}$ \\
\hline Dv & Diverging angle $=5.93^{\circ}$ & $0.15,0.20,0.25$ & $0.027,0.032,0.037$, & $0.211-$ & $0.471-$ \\
5.93 & $(5$ m diverging reach) & $0.30,0.40,0.50$ & $0.043,0.055,0.067$ & 0.581 & 1.949 \\
\hline Dv & Diverging angle $=9.83^{\circ}$ & $0.15,0.20,0.25$ & $0.025,0.029,0.035$, & $0.192-$ & $0.440-$ \\
9.83 & $(3$ m diverging reach) & $0.30,0.40,0.50$ & $0.041,0.053,0.065$ & 0.544 & 1.862 \\
\hline Dv & Diverging angle $=14.57^{\circ}$ & $0.15,0.20,0.25$ & $0.024,0.028,0.033$, & $0.189-$ & $0.426-$ \\
14.57 & $(3$ m diverging reach) & $0.30,0.40,0.50$ & $0.040,0.051,0.062$ & 0.517 & 1.801 \\
\hline Pri & Prismatic & $0.15,0.20,0.25$ & $0.035,0.043,0.055$, & $0.713-$ & $0.211-$ \\
560 & (Floodplain 560mm) & $0.30,0.40,0.50$ & $0.069,0.109,0.176$ & 0.786 & 1.229 \\
\hline \multicolumn{7}{|c|}{${ }^{(1)}$ At x=14.5m, 15.5m and 16m for Dv5.93, Dv9.83, and Dv14.57 respectively, } \\
\hline
\end{tabular}

\section{Experimental results}

Based on time and depth-averaged velocities $\left(U_{d}\right)$ [4], the velocity distributions of all the diverging series are compared at midlength of the diverging reach. For this purpose, normalised velocities are obtained by dividing the velocities $U_{d}$ by the total discharge $Q$. Figure $2(\mathrm{a}, \mathrm{b}, \mathrm{c})$ represents the velocity distribution for the central section (floodplain width $560 \mathrm{~mm}$ ) of the diverging compound channels and Fig. (2d) represents the velocity distribution in the prismatic compound channel for $560 \mathrm{~mm}$ floodplain width. Figure 2 shows that the velocity distribution is almost the same in the main channel for a given relative flow depth $(\mathrm{Dr}=(\mathrm{H}-\mathrm{h}) / \mathrm{H}))$. Differences are observed on the floodplains, due to larger discharge $\left(\mathrm{Q}=0.065 \mathrm{~m}^{3} / \mathrm{s}\right)$ to a lower floodplain velocity. 


\section{River Flow 2018}
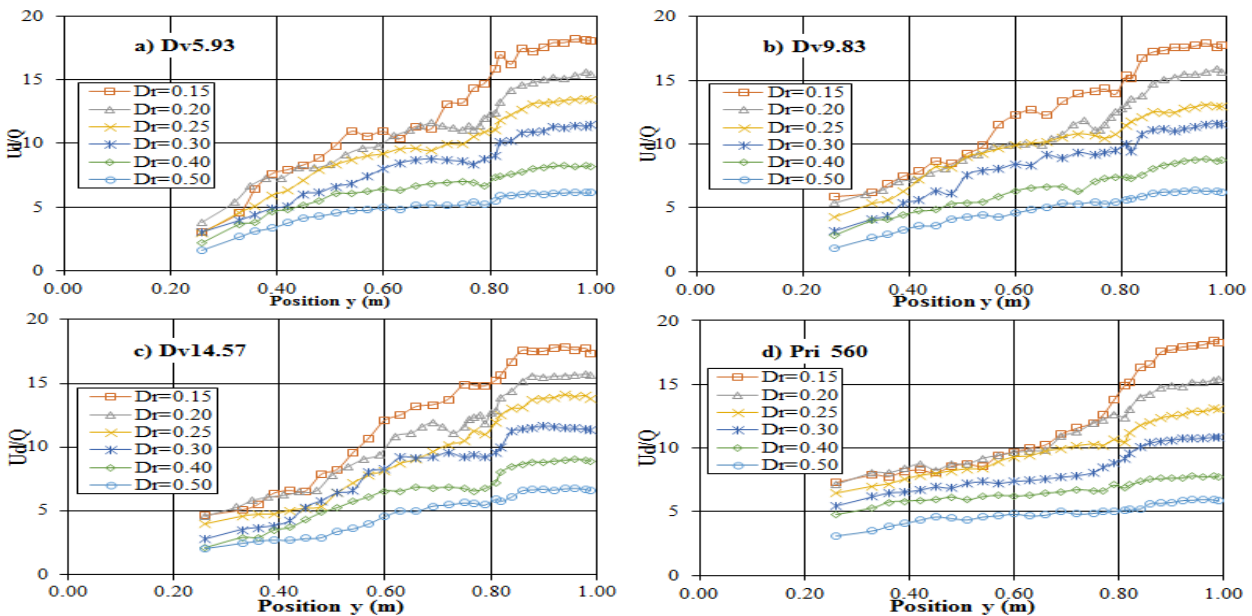

Fig. 2. Depth-averaged velocity is normalized by the total discharge, (a-c) at the mid of the diverging length and (d) at $\mathrm{x}=16 \mathrm{~m}$ for prismatic compound channel
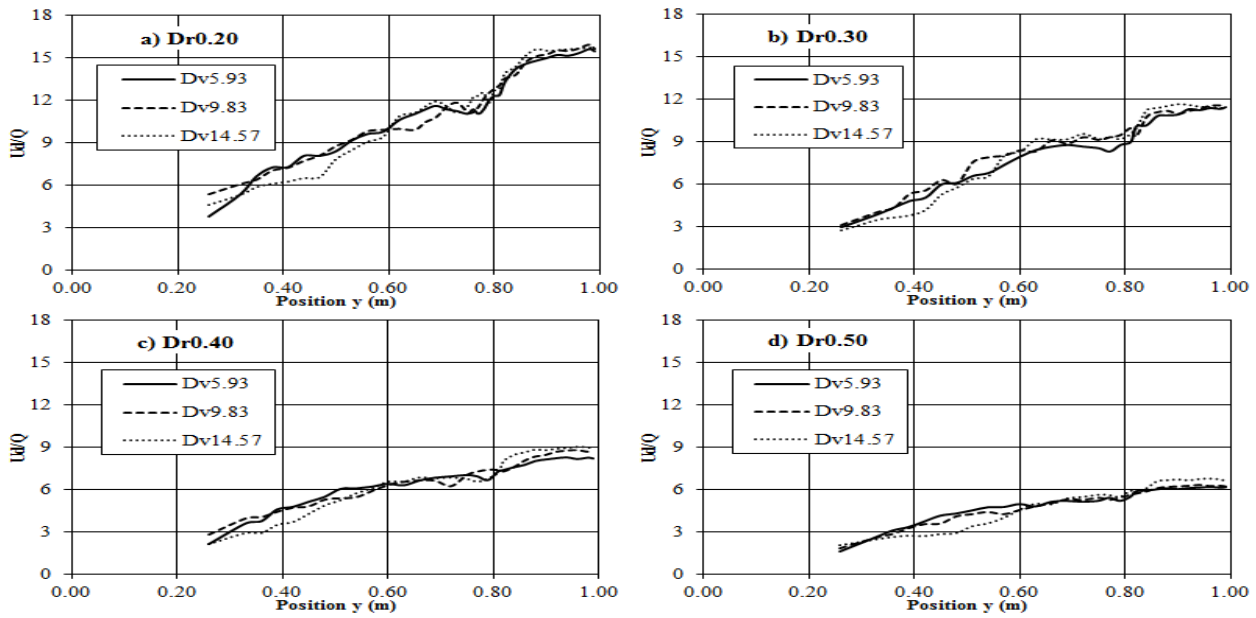

Fig. 3. Depth-averaged velocity is normalized by the total discharge, at the mid of the diverging length for different relative flow depth, a) $\mathrm{Dr}=0.20, \mathrm{~b}$ ) $\mathrm{Dr}=0.30, \mathrm{c}) \mathrm{Dr}=0.40$, and d) $\mathrm{Dr}=0.50$
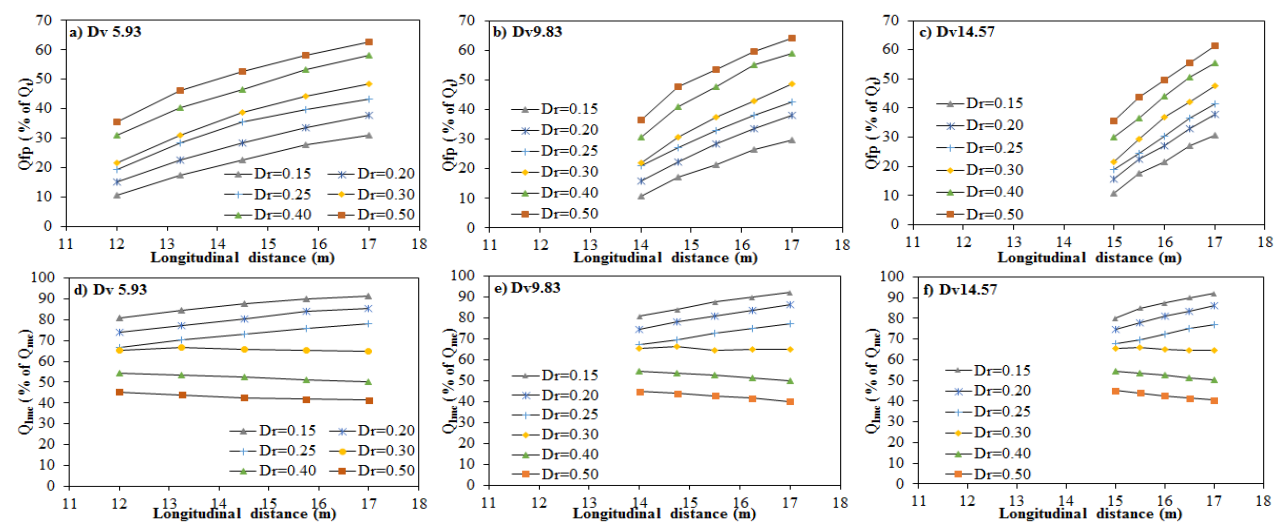

Fig. 4. In steam wise direction for the diverging portion, percentage of flow (a-c) at floodplain computed from the total flow, (d-f) at lower main channel computed from the main channel flow 
The main channel velocities are more uniform for Dv9.83 and Dv14.57 series than for Dv5.93: this could indicate lower secondary currents for the shorter diverging reach, where the mass transfer could be too large for such currents to fully develop. Figure 3 (a-d) represents the velocity distribution normalized by the total discharge for four different $\mathrm{Dr}$ values i.e., $0.2,0.3,0.4$ and 0.5 . It is observed that the $\mathrm{Ud} / \mathrm{Q}$ value at the main channel for higher angle 14.57 is slightly more than other diverging angles for all relative flow depth cases but less in floodplains due to the shorter diverging reach of $2 \mathrm{~m}$ (Fig. 1c). This causes flow separation in the diverging portion which in turns slows down the velocity at floodplains. The progressive flow expansion out of the main channel is interestingly represented by the discharge distribution between subsections (Fig. 4) as also observed by Bousmar et al. [2]. Figure 4 shows the floodplain discharge expressed as a percentage of the total discharge. This depicts clearly the progressive intrusion of the flow to the floodplain. In Fig. 4 (d-f) the percentage of flow in lower main channel increases in the streamwise direction due to higher velocity in the main channel for low value of relative flow depth i.e., $0.15,0.20$ and 0.25 which causes less mass transfer from main channel to floodplain whereas for $\mathrm{Dr}=0.3,0.4$ and 0.5 , the velocity at lower main channel decreases causing less conveyance of the main channel. The other reason for higher velocity in the lower main channel is due to the channel geometry in which the flow is allowed in the compound section and then the floodplain diverge.

\section{Improved independent subsection method (Improved-ISM)}

To determine the magnitudes of flow and velocities in the upper and lower main channels the ISM needs to be improved. Therefore, instead of three subsections of classical ISM, four subsections (left floodplain, upper main channel, lower main channel and right floodplain) are created in improved ISM according to the vertical and horizontal division lines that corresponds to vertical interface between main channel and floodplain and horizontal interface between upper and lower main channels respectively [7].The improved ISM consists in a set of four coupled 1D momentum equations for four subsections and a mass conservation equation for the total cross-section. Like classical ISM, mass and momentum exchanges at the interface between the subsections are considered explicitly. Figure 5 shows the schematic diagram of compound channel subsections. Velocities at left floodplain, upper main channel, right floodplain and lower main channel represented by $U_{l}$, $U_{2}, U_{3}$, and $U_{4}$ respectively and the floodplain flow depth represented by $h_{f p}\left(=h_{12}=h_{23}\right)$.

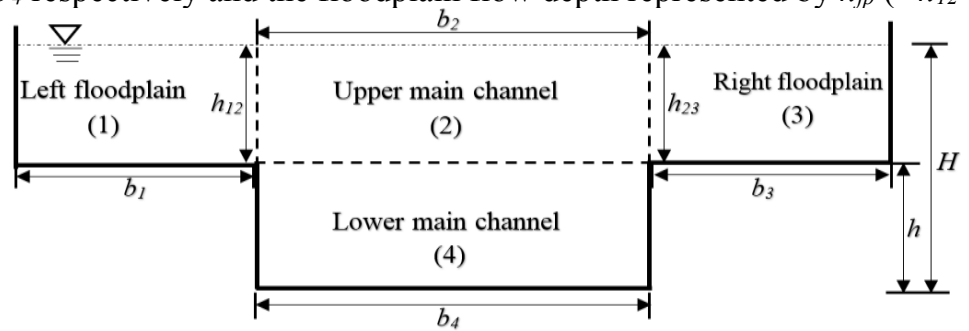

Fig. 5. Division of compound channel

In this division process, we have distinguished the incoming and outgoing lateral flows, These mass flows are naturally exclusive: in a subsection, the mass is either incoming or outgoing. The equations were written in such a way that $q_{i n}$ and $q_{o u t}$ are always positive. Now, we will consider algebraic lateral flows: $q_{12}$ will be the exchange rate from the FP left to the MC, $q_{32}$ the exchange rate from the FP right to the upper MC and $q_{42}$ the exchange rate from the LMC to the UMC. These three flows will be positive if mass leaves from the 
FP and UMC, and negative, if the mass enters the FP and UMC. In this way, the equations of continuity per floodplains, upper and lower main channels can be written as :

$$
\begin{gathered}
\frac{d Q_{1}}{d x}=-q_{12}, \frac{d Q_{3}}{d x}=-q_{32}, \frac{d Q_{4}}{d x}=-q_{42}, \text { and } \frac{d Q_{2}}{d x}=q_{12}+q_{32}+q_{42} \\
\frac{d Q_{1}}{d x}+\frac{d Q_{2}}{d x}+\frac{d Q_{3}}{d x}+\frac{d Q_{4}}{d x}=0
\end{gathered}
$$

1D momentum equations for the four subsections are :

For left floodplain $\quad\left(1-\frac{U_{1}^{2}}{g h_{12}}\right) \frac{d h_{12}}{d x}=S_{0}-S_{f_{1}}+\frac{U_{1}^{2}}{g b_{1}} \frac{d b_{1}}{d x}+\frac{\tau_{12} \cdot h_{12}}{\rho g A_{1}}+\frac{q_{12} \cdot\left(2 U_{1}-U_{\text {int.12 }}\right)}{g A_{1}}$

For right floodplain

$$
\left(1-\frac{U_{3}^{2}}{g h_{23}}\right) \frac{d h_{32}}{d x}=S_{0}-S_{f_{3}}+\frac{U_{3}^{2}}{g b_{3}} \frac{d b_{3}}{d x}+\frac{\tau_{32} \cdot h_{32}}{\rho g A_{3}}+\frac{q_{32} \cdot\left(2 U_{3}-U_{\text {int. } 32}\right)}{g A_{3}}
$$

For lower main channel

$$
\left(1-\frac{U_{4}^{2}}{g h}\right) \frac{d h}{d x}=S_{0}-S_{f_{4}}+\frac{U_{4}^{2}}{g b_{4}} \frac{d b_{4}}{d x}+\frac{\tau_{42} \cdot b_{4}}{\rho g A_{4}}+\frac{q_{42} \cdot\left(2 U_{4}-U_{\mathrm{int}, 42}\right)}{g A_{4}}
$$

For upper main channel

$$
\begin{aligned}
& \left(1-\frac{U_{2}^{2}}{g h_{12}}\right) \frac{d h_{12}}{d x}=S_{0}-S_{f_{2}}+\frac{U_{2}^{2}}{g B_{2}} \frac{d B}{d x}-\frac{\tau_{12} \cdot h_{12}}{\rho g A_{2}}-\frac{\tau_{32} \cdot h_{32}}{\rho g A_{2}}-\frac{\tau_{42} \cdot b_{4}}{\rho g A_{2}}+\ldots \\
& \ldots-\frac{q_{12} \cdot\left(2 U_{2}-U_{\text {int.12 }}\right)}{g A_{2}}-\frac{q_{32} \cdot\left(2 U_{2}-U_{\text {int. } .32}\right)}{g A_{2}}-\frac{q_{42} \cdot\left(2 U_{2}-U_{\text {in } .42}\right)}{g A_{2}}
\end{aligned}
$$

The interfacial velocity between the zone 1 and the 2 being noted as $U_{\text {int.12, }}$, that between the 3 and $2, U_{\text {int.32, }}$, and between the 4 and $2, U_{\text {int. } 42}$, and the shear at the left interface, $\tau_{12}$, at the right interface $\tau_{32}$ and at lower and upper MC interface is $\tau_{42}$. The interfacial velocity between LMC and UMC is computed by the relation similar to Proust [1], where the contribution of velocities of UMC and LMC to the interfacial velocity $U_{\text {int.42 }}$ is weighted by its depth of the subsection and presented as follows :

$$
U_{h . \text { int } \text { Model }}=\frac{h}{h+3.5 h_{12}} U_{2}+\frac{3.5 h}{h_{b}+3.5 h_{12}} U_{4}
$$

Figure 6 (a) shows the location of horizontal interfacial velocity between the UMC and LMC and the value can be modelled by Eq. (7). Figure 6 (b) shows the horizontal $U_{\text {int. }}$ velocity model for Dv5.93 with Dr 0.4. Modelled values show a good match with measured values and thus used in the simulation of improved ISM to compute the discharge for LMC and UMC. The details of improved ISM method is described in Das [15].
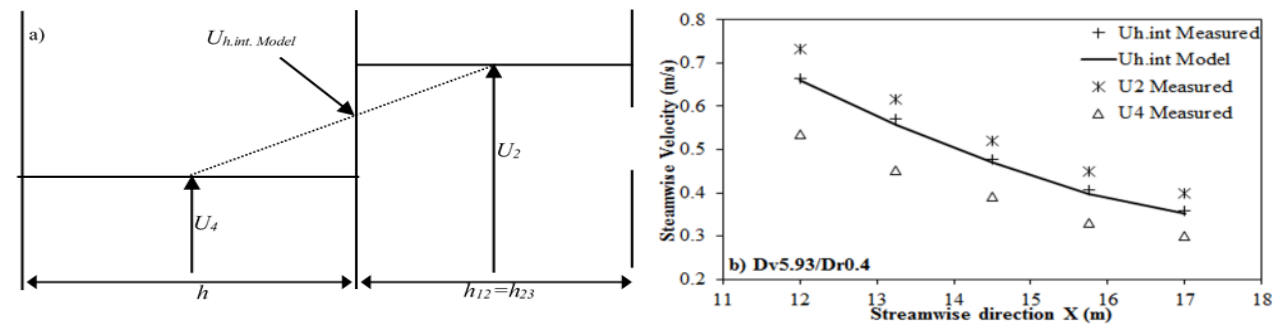

Fig. 6. Interfacial velocity: a) upper main channel and lower main channel, b) streamwise width averaged velocity at the horizontal interface Uh.int. Modelled vs experimental

\subsection{Differential equation modelling}

Equations (2), (3), (4), (5) and (6), can be written as an ordinary differential equation system (8), where coefficients $a_{1}, a_{2}, a_{3}, a_{4}, a_{5}, b_{1}, b_{2}, c_{1}, c_{4}, d_{1}, d_{5}, e_{1}, e_{2}, e_{4}$, and $e_{5}$ are non- 
linear functions of velocities $U_{1}, U_{2}, U_{3}, U_{4}$, and floodplain water depth $h_{f p}\left(=h_{12}=h_{23}\right)$, geometrical parameters and subsection Manning's roughness [15].

$$
\left\{\begin{array}{l}
a_{1} \frac{d h_{1}}{d x}+a_{2} \frac{d U_{1}}{d x}+a_{3} \frac{d U_{2}}{d x}+a_{4} \frac{d U_{3}}{d x}+a_{5} \frac{d U_{4}}{d x}=0 \\
b_{1} \frac{d h_{1}}{d x}+b_{2} \frac{d U_{1}}{d x}=0 \\
c_{1} \frac{d h_{1}}{d x}+c_{4} \frac{d U_{3}}{d x}=0 \\
d_{1} \frac{d h_{1}}{d x}+d_{5} \frac{d U_{4}}{d x}=0 \\
e_{1} \frac{d h_{1}}{d x}+e_{2} \frac{d U_{1}}{d x}+e_{4} \frac{d U_{3}}{d x}+e_{5} \frac{d U_{4}}{d x}=0
\end{array}\right.
$$

The improved ISM consists thus in solving both flow depth and subsection velocities simultaneously. The MATLAB solving procedure ODE45 for ordinary differential equations was used: it is based on a Runge-Kutta explicit scheme of 4th or 5th order. For subcritical flows, the ISM is solved iteratively: the upstream experimental discharge distribution and a tentative upstream water level are given. The downstream water level results from the computation. The upstream level is then adjusted until the suitable downstream level is obtained.

\subsection{Improved ISM simulations}

The improved ISM is simulated by considering the total momentum (both turbulence and mass) transfer between the subsections. The results obtained from the simulation for some selected flow depths and diverging angles are shown in Table 2. The divided channel method has also been used to compute the floodplain discharge and Strickler equation has been used to compute the lower main channel discharge. From Table 2, it is noticed that the floodplain flow percentage obtained from the improved ISM (IISM) give a good match with computed $\%$ Qfp ( $\%$ of total discharge). Further, the improved ISM is able to compute the lower main channel discharge. For lower main channel discharge distribution, the IISM shows good accuracy with experimental value as compared to the value obtained from Strickler equation. Figure 7 (a-c) shows the lower main channel flow percentage from the main channel flow for Dv5.93/Dr0.25, Dv9.83/Dr0.5, and Dv14.57/Dr0.4 respectively. The increase of \%Qlmc (Fig. 7a) and decrease \%Qlmc (Fig. 7b and 7c) along the streamwise direction is due to the effect of relative flow depths as it has been discussed in section 3 .

Table 2. Comparison of Qfp ( $\%$ of total discharge) and $\% \mathrm{Qlmc}(\%$ of main channel discharge) by different methods

\begin{tabular}{|c|c|c|c|c|c|c|c|c|c|c|}
\hline \multirow{2}{*}{ Series } & \multirow{2}{*}{ Dr } & \multirow{2}{*}{$\mathbf{X}(\mathbf{m})$} & \multicolumn{4}{|c|}{ Qfp(\% Q total) } & \multicolumn{4}{c|}{ QImc(\% Q mc) } \\
\cline { 4 - 12 } & & & EXP & DCM & ISM & IISM & EXP & $\begin{array}{c}\text { Str. } \\
\text { Eq. }\end{array}$ & ISM & IISM \\
\hline Dv & 0.15 & 14.50 & 22.68 & 26.5 & 22.8 & 22.91 & 87.61 & 90.6 & - & 86.68 \\
\cline { 2 - 11 } 5.93 & 0.50 & 14.50 & 52.56 & 55.3 & 53.2 & 53.16 & 52.64 & 55.6 & - & 51.85 \\
\hline Dv & 0.25 & 15.50 & 32.98 & 36.4 & 33.2 & 33.15 & 72.49 & 75.6 & - & 73.66 \\
\cline { 2 - 11 } 9.83 & 0.40 & 16.25 & 55.30 & 58.2 & 55.8 & 55.74 & 51.33 & 54.7 & - & 52.64 \\
\hline $\begin{array}{c}\text { Dv } \\
14.57\end{array}$ & 0.30 & 16.00 & 36.73 & 40.1 & 36.9 & 37.26 & 64.82 & 67.9 & - & 65.13 \\
\cline { 2 - 10 } & 0.50 & 16.50 & 55.60 & 58.8 & 56.1 & 55.96 & 41.43 & 44.0 & - & 41.81 \\
\hline
\end{tabular}

X- longitudinal distance (m), DCM-Divided channel method, Str. Eq.- Strickler Equation, EXPexperimental data, ISM- classical ISM (Proust 2006), IISM-Improved ISM 

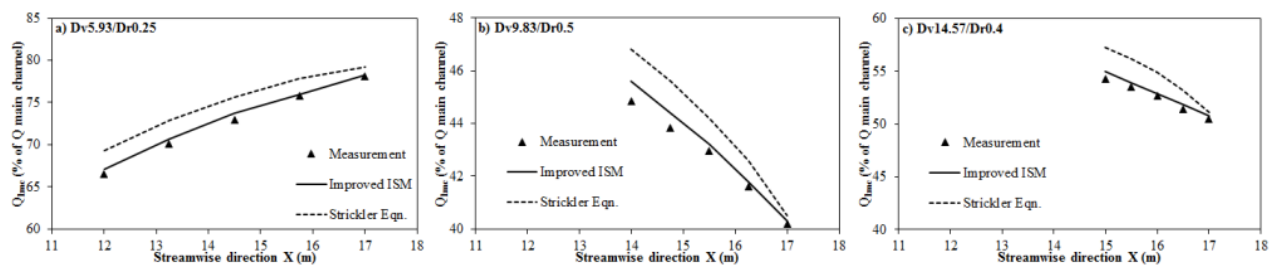

Fig. 7. Comparison of lower main channel flow distribution (\% of main channel flow)

\section{Conclusions}

Experiments have been performed in the compound channel with three different diverging angles where the floodplain diverge from the prismatic floodplain. The depth-averaged velocity variation in the main channel for lower diverging length channel i.e., Dv9.83 (3m diverging length) and Dv14.57 (2m) are more uniform compared to Dv 5.93(5m). The large variation in the floodplain discharge is observed due to mass and turbulence transfer from the main channel to the floodplain. In the diverging portion for relative flow depth $0.15,0.2$ and 0.25 the percentage of flow in the lower main channel increases along the streamwise direction and for $0.3,0.4$ and 0.5 relative flow depth, it is decreasing. Horizontal interfacial velocity between upper and lower main channel is modelled by considering flow depth in the upper main channel and bank full depth of the main channel. Accounting of momentum exchange in the horizontal shear layer region between the upper and lower main channels and vertical shear layer region between upper main channel and floodplains in the improved ISM make the model to predict lower main channel discharge along with the flow depths and velocities at different subsections of the channel and show a good match with the experimental findings.

This work was supported by the UGC UKIERI Research project [Ref no. UGC-2013 14/017].

\section{References}

1. S. Proust, Doctoral dissertation, INSA de Lyon (2005).

2. D. Bousmar, S. Proust, Y. Zech, In River Flow 2006, 323-332 (2006).

3. H. A. Yonesi, M. H. Omid, S. A. Ayyoubzadeh, J Civil Eng Urban, 3, 6, 342-356 (2013).

4. B. S. Das, K. K. Khatua, K. Devi, Arabian J. for Sci. and Eng., 42(3), pp.1305-1319, (2017).

5. B. S. Das, K. K. Khatua, Arabian J. for Sci. and Eng., 1-15 (2018) (in press).

6. B. S. Das, K. K. Khatua, ISH J.of Hyd. Eng., 1-14 (2018) (in press).

7. D.W. Knight, J.D. Demetriou (1983), J. of Hyd. Eng., 109, 8, 1073-1092 (1983).

8. P.R. Wormleaton, J. Allen, P. Hadjipanos, J.of Hyd. Div., 108, 9, 975-994 (1982).

9. K. Devi, K. K. Khatua, B. S. Das, J.R. Khuntia, ISH J. of Hyd. Eng., 23, 2, 187-194 (2017).

10. K. Yang, X. Liu, S. Cao, E. Huang, (2013). J. of Hyd. Eng., 140, 4, 0601400, (2013).

11. S. Proust, D. Bousmar, N. Riviere, A. Paquier, Y. Zech, Y., WRR, 45, 12 (2009).

12. S. Proust, D. Bousmar, N. Rivière, A. Paquier, Y. Zech, Adv. in water Resour., 33, 1, 1-16 (2010).

13. S. Proust, C. Berni, .... E. Mignot, In 3rd Euro. Conf. on Flood Risk Mgmt. (pp. 12-p), (2016).

14. B. S. Das, K. K. Khatua, J.of Hyd. Eng., ASCE, (2018) (in production).

15. B. S. Das, Doctoral dissertation, NIT Rourkela (2018) (to be published). 